\title{
A Caputo Two-Point Boundary Value Problem: Existence, Uniqueness and Regularity of a Solution
}

\author{
Stynes M. \\ Received May 19, 2016
}

\begin{abstract}
.
A two-point boundary value problem on the interval $[0,1]$ is considered, where the highest-order derivative is a Caputo fractional derivative of order $2-\delta$ with $0<\delta<1$. A necessary and sufficient condition for existence and uniqueness of a solution $u$ is derived. For this solution the derivative $u^{\prime}$ is absolutely continuous on $[0,1]$. It is shown that if one assumes more regularity - that $u$ lies in $C^{2}[0,1]$ - then this places a subtle restriction on the data of the problem.
\end{abstract}

Keywords: fractional derivative, boundary value problem, existence, uniqueness, regularity

For citation: Stynes M., "A Caputo two-point boundary value problem: existence, uniqueness and regularity of a solution", Modeling and Analysis of Information Systems, 23:3 (2016), 370-376.

On the authors:

Stynes M., Beijing Computational Science Research Center, Haidian District,

Beijing 100193, China, e-mail m.stynes@csrc.ac.cn

\section{Introduction}

Fractional derivatives are very fashionable at present: they are used in many recent models to give results that seem to be unattainable by classical integer-order derivatives. Consequently there is a huge amount of current research activity in the area of numerical methods for the solution of differential equations that involve fractional-order derivatives. Unfortunately, many papers analysing numerical methods for fractionalderivative problems neglect to discuss existence, uniqueness and regularity of the the solution to the problem they are solving - these fundamental and crucial properties are simply assumed to be true!

In the present paper, which is partly based on [5], we consider a Caputo two-point boundary value that is defined in Section 1.. This problem models superdiffusion of particle motion when convection is present; see [3, Section 1] and its references. In Section 2. we shall derive a necessary and sufficient condition for existence and uniqueness of a solution to this problem in a certain space of functions that lies between $C^{1}[0,1]$ and $C^{2}[0,1]$. Then in Section 3. we show that if one assumes that $u$ lies in $C^{2}[0,1]$ i.e., one assumes more regularity of the solution - then this places a subtle restriction on the data of the problem. 
Notation. All functions are real valued. $C(I)$ comprises those functions that are continuous on an interval $I$, and $C^{k}(I)$ denotes the space of functions defined on $I$ whose derivatives up to order $k$ lie in $C(I)$, for $k=1,2, \ldots$ We follow the convention that $C^{0}(I)=C(I)$. Denote by $L_{1}[0,1]$ the standard Lebesgue space of integrable functions defined almost everywhere on $[0,1]$.

\section{The Caputo two-point boundary value problem}

The following definitions are needed to describe our boundary value problem.

For $r \in \mathbb{R}$ with $r>0$, and all $g \in L_{1}[0,1]$, the Riemann-Liouville fractional integral operator $J^{r}$ of order $r$ is defined by

$$
\left(J^{r} g\right)(x)=\left[\frac{1}{\Gamma(r)} \int_{t=0}^{x}(x-t)^{r-1} g(t) d t\right] \quad \text { for } \quad 0 \leq x \leq 1
$$

Let the parameter $\delta$ satisfy $0<\delta<1$. Let $g \in C^{1}[0,1]$ with $g^{\prime}$ absolutely continuous on $[0,1]$. Then the Caputo fractional derivative $D_{*}^{2-\delta} g$ of order $2-\delta$ is defined for almost all $x \in(0,1)$ (see, e.g., [4, Theorem 2.1]) by

$$
D_{*}^{2-\delta} g(x):=\left(J^{\delta} g^{\prime \prime}\right)(x)=\frac{1}{\Gamma(\delta)} \int_{t=0}^{x}(x-t)^{\delta-1} g^{\prime \prime}(t) d t \quad \text { for } \quad 0<x \leq 1 .
$$

Since the integral in $D_{*}^{2-\delta} g(x)$ is associated with the point $x=0$, many authors write instead $D_{* 0}^{2-\delta} g(x)$, but for simplicity of notation we omit the subscript 0 .

We shall consider the two-point boundary value problem

$$
-D_{*}^{2-\delta} u(x)+b(x) u^{\prime}(x)+c(x) u(x)=f(x) \text { for } x \in(0,1),
$$

subject to the boundary conditions

$$
\begin{aligned}
& u(0)-\alpha_{0} u^{\prime}(0)=\gamma_{0}, \\
& u(1)+\alpha_{1} u^{\prime}(1)=\gamma_{1},
\end{aligned}
$$

where the constants $\alpha_{0}, \alpha_{1}, \gamma_{0}, \gamma_{1}$ and the functions $b, c$ and $f$ are given. We assume that $b, c, f \in C^{q}[0,1]$ for some integer $q \geq 1$.

Remark 1. If one also assumes that $c \geq 0$ on $[0,1], \alpha_{0} \geq 1 /(1-\delta)$ and $\alpha_{1} \geq 0$, then (3) satisfies a comparison/maximum principle, from which existence and uniqueness of the solution $u$ of (3) follows; see [Y]. But if the Robin boundary condition at $x=0$ is replaced by a Dirichlet boundary condition, then the comparison/maximum principle may no longer be true: a counterexample is given in [7, Example 2.4].

A more general class of boundary value problems is considered in [6]. Numerical methods for the solution of (3) are presented and analysed rigorously in, for instance, $[3,5,6,7]$.

The present paper will discuss some theoretical aspects of (3): existence, uniqueness and regularity of solutions. Existence and uniqueness of a solution using the space 
$C^{q, \delta}(0,1]$ defined below was proved in [5] by means of a reformulation in terms of Volterra integral equations of the second kind, under the additional hypotheses that

$$
c \geq 0, \alpha_{0} \geq 0 \text { and } \alpha_{1} \geq 0 .
$$

We shall use the same Volterra reformulation here but interpret its conclusions in a more general way that yields conditions on the data that are necessary and sufficient for existence and uniqueness of a solution to (3).

When discussing solutions of $(3)$, the following setting is natural $[1,8]$. Let $C^{q, \delta}(0,1]$ be the space of all functions $y \in C[0,1] \cap C^{q}(0,1]$ such that

$$
\|y\|_{q, \delta}:=\sup _{0<x \leq 1}|y(x)|+\sum_{k=1}^{q} \sup _{0<x \leq 1}\left[x^{k-(1-\delta)}\left|y^{(k)}(x)\right|\right]<\infty .
$$

That is, one has $|y(x)| \leq C$ and $\left|y^{(k)}(x)\right| \leq C x^{(1-\delta)-k}$ for $k=1, \ldots, q$. By [8], $C^{q, \delta}(0,1]$ is a Banach space. Note that $C^{q}[0,1] \subset C^{q, \delta}(0,1]$.

Define the space of functions

$$
C_{1}^{q, \delta}(0,1]:=\left\{y \in C^{1}[0,1] \cap C^{q+1}(0,1]: y^{\prime} \in C^{q, \delta}(0,1]\right\} .
$$

We are interested only in those solutions $u$ of (3) that lie in $C_{1}^{q, \delta}(0,1]$. This is a reasonable class of candidates for solutions of $(3)$, since then $D_{*}^{2-\delta} u$ is defined everywhere in $(0,1]$ by Lemma 1 below, and as we shall see in Section 3., imposing more regularity on $u^{\prime \prime}$ by requiring $u \in C^{2}[0,1]$ would lead to certain difficulties.

Lemma 1. Let $y \in C_{1}^{q, \delta}(0,1]$. Then $D_{*}^{2-\delta} y(x)$ is defined for all $x \in(0,1]$.

Proof. Let $x \in(0,1]$. Then $D_{*}^{2-\delta} y(x)=(1 / \Gamma(\delta)) \int_{t=0}^{x}(x-t)^{\delta-1} y^{\prime \prime}(t) d t$, provided this integral exists. Invoking the hypothesis that $y \in C_{1}^{q, \delta}(0,1]$, one has

$$
\frac{1}{\Gamma(\delta)} \int_{t=0}^{x}\left|(x-t)^{\delta-1} y^{\prime \prime}(t)\right| d t \leq \frac{C}{\Gamma(\delta)} \int_{t=0}^{x}(x-t)^{\delta-1} t^{-\delta} d t=C \Gamma(1-\delta)
$$

by a standard formula for Euler's Beta function [2, Theorem D.6]. Hence the integral exists in the Lebesgue sense, i.e., $D_{*}^{2-\delta} y(x)$ is defined.

Example 1. Consider the simple problem $D_{*}^{2-\delta} u=\Gamma(3-\delta)$ on $(0,1), u(0)=0, u(1)=1$. From [2, pages 55 and 193] it is easy to see that the unique solution $u$ of this problem is $u(x)=x^{2-\delta}$. Hence $u \in C_{1}^{m, \delta}(0,1]$ for any positive integer $m$, but $u \notin C^{2}[0,1]$.

The regularity of the solution of Example 1 is typical of solutions to the general boundary value problem (3).

\section{Existence and uniqueness of a solution}

Define the Volterra operator $L$ by

$$
L z(x)=z(x)-\frac{1}{\Gamma(1-\delta)} \int_{t=0}^{x}(x-t)^{-\delta}\left[b(t) z(t)+c(t) \int_{0}^{t} z(s) d s\right] d t \quad \text { for } 0 \leq x \leq 1 .
$$


It is shown in the proof of $\left[5\right.$, Lemma 2.1] that $L: C^{q, \delta}(0,1] \rightarrow C^{q, \delta}(0,1]$ is a compact operator.

Consider now two Volterra integral equations of the second kind: for $0 \leq x \leq 1$,

$$
L v(x)=\frac{1}{\Gamma(1-\delta)} \int_{t=0}^{x}(x-t)^{-\delta}\left[b(t)+\left(t+\alpha_{0}\right) c(t)\right] d t
$$

and

$$
L w(x)=\frac{1}{\Gamma(1-\delta)} \int_{t=0}^{x}(x-t)^{-\delta}\left[\gamma_{0} c(t)-f(t)\right] d t
$$

From [5, Lemma 4.1], the solutions $v$ and $w$ are well defined and lie in $C^{q, \delta}(0,1]$.

Theorem 1 (Existence and uniqueness of a solution to (3)). Set

$$
\theta=\alpha_{0}+\alpha_{1}[1+v(1)]+\int_{0}^{1}[1+v]
$$

1. If $\theta \neq 0$, then (3) has a unique solution

$$
u(x)=\gamma_{0}+\mu \alpha_{0}+\int_{t=0}^{x}[\mu(1+v(t))+w(t)] d t
$$

with $u \in C_{1}^{q, \delta}(0,1]$, where

$$
\mu=\frac{\gamma_{1}-\gamma_{0}-\alpha_{1} w(1)-\int_{0}^{1} w}{\theta}
$$

2. If $\theta=0$, then (3) has either no solution or infinitely many solutions in $C_{1}^{q, \delta}(0,1]$.

Proof. The analysis of [5] shows that for any $\mu \in \mathbb{R}$ the function

$$
u(x)=u(0)+\mu x+\int_{0}^{x}(\mu v+w)(t) d t
$$

lies in $C_{1}^{q, \delta}(0,1]$ and will satisfy the differential equation (3a) and the boundary condition (3b); it is also shown in [5] that if a function $u \in C_{1}^{q, \delta}(0,1]$ satisfies (3a) and (3b), then $u$ satisfies (8). Thus it remains only to choose $\mu$ in (8) such that $u$ satisfies the boundary condition (3c): $u(1)+\alpha_{1} u^{\prime}(1)=\gamma_{1}$.

Using (8) and eliminating $u(0)$ by means of $(3 \mathrm{~b})$, one has

$$
\begin{aligned}
u(1)+\alpha_{1} u^{\prime}(1) & =u(0)+\mu+\alpha_{1}[\mu+\mu v(1)+w(1)]+\int_{0}^{1}(\mu v+w) \\
& =\gamma_{0}+\alpha_{0} \mu+u(0)+\mu+\alpha_{1}[\mu+\mu v(1)+w(1)]+\int_{0}^{1}(\mu v+w) \\
& =\gamma_{0}+\mu \theta+\alpha_{1} w(1)+\int_{0}^{1} w .
\end{aligned}
$$


If $\theta \neq 0$, then the unique choice of $\mu$ given by (7) yields $u(1)+\alpha_{1} u^{\prime}(1)=\gamma_{1}$ and the solution of (3) is then specified by (8).

If $\theta=0$, there are two possibilities: if $\gamma_{0}+\alpha_{1} w(1)+\int_{0}^{1} w \neq \gamma_{1}$ then the boundary condition (3c) cannot be satisfied and (3) has no solution, while if $\gamma_{0}+\alpha_{1} w(1)+\int_{0}^{1} w=\gamma_{1}$, then the boundary condition (3c) is satisfied for any choice of $\mu$ and we have infinitely many solutions given by (8) where $\mu \in \mathbb{R}$ is arbitrary.

In [5, Theorem 4.1] it was shown that when (4) is satisfied, one then has $\theta>0$ and consequently (3) has a unique solution, but the more general situation described in Theorem 1 was not discussed.

\section{Effect of assuming that $u \in C^{2}[0,1]$}

In Sections 1. and 2., solutions of (3) lying in the space $C_{1}^{q, \delta}(0,1]$ were considered. These solutions are smooth on $(0,1]$ but typically much less smooth on the closed interval $[0,1]$. The present section examines the effect of assuming that the solution $u$ lies not just in $C_{1}^{q, \delta}(0,1]$ but in the space $C^{2}[0,1]$ for which $u^{\prime \prime}$ is bounded on $[0,1]$. We show that with this assumption, the class of problems under consideration is restricted more severely than one would expect.

Higher regularity of solutions on the closed interval [0,1] is commonly assumed in numerical analyses of fractional-derivative problems, but many researchers seem unaware of the consequences of this assumption. We describe here what $u \in C^{2}[0,1]$ implies for our problem (3); our results can easily be generalised to Caputo differential equations (boundary value problems and initial-value problems) of any order.

The crucial observation is the following result (see, e.g., [2, Lemma 3.11]), whose short elementary proof we include for completeness.

Lemma 2. Let $g \in C^{2}[0,1]$. Then

$$
\lim _{x \rightarrow 0^{+}} D_{*}^{2-\delta} g(x)=0 .
$$

Proof. For any $x \in(0,1)$,

$$
D_{*}^{2-\delta} g(x)=\frac{1}{\Gamma(\delta)} \int_{t=0}^{x}(x-t)^{\delta-1} g^{\prime \prime}(t) d t .
$$

But $g \in C^{2}[0,1]$ implies that $\left|g^{\prime \prime}(t)\right| \leq C$ for $0 \leq t \leq 1$ and some constant $C$. Hence

$$
\left|D_{*}^{2-\delta} g(x)\right| \leq \frac{C}{\Gamma(\delta)} \int_{t=0}^{x}(x-t)^{\delta-1} d t=\frac{C x^{\delta}}{\Gamma(\delta+1)} \rightarrow 0 \text { as } x \rightarrow 0^{+} .
$$

Remark 2. The converse of Lemma 2 is false. For suppose $g(x)=x^{2-\beta}$ with $0<\beta<\delta$. Then $g \in C_{1}^{q, \delta}(0,1]$ but $g \notin C^{2}[0,1]$, and

$$
D_{*}^{2-\delta} g(x)=\frac{(2-\beta)(1-\beta)}{\Gamma(\delta)} \int_{t=0}^{x}(x-t)^{\delta-1} t^{-\beta} d t=\frac{\Gamma(3-\beta)}{\Gamma(1+\delta-\beta)} x^{\delta-\beta} \rightarrow 0 \text { as } x \rightarrow 0^{+},
$$

where we used the standard formula for Euler's Beta function [2, Theorem D.6] to evaluate the integral. 
Remark 3. By imitating the calculation of Remark 2, one can replace the hypothesis $g \in C^{2}[0,1]$ of Lemma 2 by the weaker assumption that $g \in C_{1}^{2, \beta}(0,1]$ for some $\beta \in(0, \delta)$.

Assume now that (3) has a solution $u \in C^{2}[0,1]$. Then by Lemma 2 we can apply $\lim _{x \rightarrow 0^{+}}$to $(3 \mathrm{a})$, obtaining

$$
b(0) u^{\prime}(0)+c(0) u(0)=f(0)
$$

One also has the boundary condition (3b); combining this with (9) yields

$$
f(0)=\left[b(0)+\alpha_{0} c(0)\right] u^{\prime}(0)+\gamma_{0} c(0) .
$$

Assume that $u$ is the unique solution of (3), i.e., assume that $\theta \neq 0$ in Theorem 1 . Then the value of $u^{\prime}(0)$ is given by (7). Thus $f$ must satisfy the equation

$$
f(0)=\frac{\left[b(0)+\alpha_{0} c(0)\right]\left[\gamma_{1}-\gamma_{0}-\alpha_{1} w(1)-\int_{0}^{1} w\right]}{\alpha_{0}+\alpha_{1}[1+v(1)]+\int_{0}^{1}[1+v]}+\gamma_{0} c(0) .
$$

As $w$ depends on $f$ by (6), the necessary condition (11) places a difficult-to-verify restriction on $f$ that is completely unnatural, and is due entirely to the arbitrary assumption that $u \in C^{2}[0,1]$.

Remark 4. In the special case where $b \equiv 0$ and $\alpha_{0}=0$, the problem (3) becomes

$$
-D_{*}^{2-\delta} u+c u=f \text { on }(0,1) \text {, with } u(0)=\gamma_{0}, u(1)+\alpha_{1} u^{\prime}(1)=\gamma_{1} .
$$

If $u \in C^{2}[0,1]$ here, we can work directly from Lemma 2 without appealing to [5]: taking the limit of the differential equation as $x \rightarrow 0^{+}$shows that

$$
f(0)=c(0) u(0)=c(0) \gamma_{0}
$$

is a necessary condition for a solution $u$ in $C^{2}[0,1]$.

The analysis of this section shows that making excessive regularity assumptions on the solution to a fractional-derivative is not only unjustified (recall Example 1) but also restricts the class of problems under consideration by imposing a condition (11) on the data that may be difficult to check in any concrete example.

\section{References}

[1] Brunner Hermann, Pedas Arvet, Vainikko Gennadi, "Piecewise polynomial collocation methods for linear Volterra integro-differential equations with weakly singular kernels", SIAM J. Numer. Anal., 39:3 (2001), 957-982, electronic.

[2] Diethelm Kai, The analysis of fractional differential equations. An application-oriented exposition using differential operators of Caputo type, Lecture Notes in Mathematics, 2004, Springer-Verlag, Berlin, 2010.

[3] Jin Bangti, Lazarov Raytcho, Pasciak Joseph, Rundell William, "Variational formulation of problems involving fractional order differential operators", Math. Comp., 84:296 (2015), 2665-2700.

[4] Kilbas Anatoly A., Srivastava Hari M., Trujillo Juan J., Theory and applications of fractional differential equations, North-Holland Mathematics Studies, 204, Elsevier Science B.V., Amsterdam, 2006. 
[5] Kopteva Natalia, Stynes Martin, "An efficient collocation method for a Caputo two-point boundary value problem", BIT, 55:4 (2015), 1105-1123.

[6] Pedas Arvet, Tamme Enn, "Piecewise polynomial collocation for linear boundary value problems of fractional differential equations", J. Comput. Appl. Math., 236:13 (2012), 3349-3359.

[7] Stynes Martin, Gracia José Luis, "A finite difference method for a two-point boundary value problem with a Caputo fractional derivative", IMA J. Numer. Anal., 35:2 (2015), 698-721.

[8] Vainikko Gennadi, Multidimensional weakly singular integral equations, Lecture Notes in Mathematics, 1549, Springer-Verlag, Berlin, 1993.

Стайнс М., "Двухточечная краевая задача Капуто: существование, единственность и регулярность решения", Моделирование и анализ информачионых систем, 23:3 (2016), 370-376.

DOI: $10.18255 / 1818-1015-2016-3-370-376$

Аннотация. Рассматривается двухточечная краевая задача на промежутке $[0,1]$, в которой старшая производная является дробной производной Капуто порядка $2-\delta$ при $0<\delta<1$. Получено необходимое и достаточное условие существования и единственности решения $u$. Производная $u^{\prime}$ этого решения оказывается абсолютно непрерывной на $[0,1]$. Показано, что предположение о большей регулярности - что $u$ принадлежит $C^{2}[0,1]$ - накладывает довольно тонкое ограничение на данные задачи.

Ключевые слова: дробная производная, краевая задача, существование, единственность, регулярность

Об авторах:

Мартин Стайнс, Пекинский Исследовательский Центр Вычислительных Наук, район Хайдянь, Пекин 100193, Китай, e-mail: m.stynes@csrc.ac.cn 